nature

biotechnology

\section{Proceed with caution}

O ften progress in biotechnology appears to be a matter of one step forward and at least an equal length step to the rear, simultaneously with several in every other direction. This is particularly true for the field of regenerative cell therapies, the focus of this issue. For the latest leap, look no further than the June 17 issue of Science, where Woo Suk Hwang and his team describe the generation of multiple human embryonic stem (ES) cell lines via somatic cell nuclear transfer. Their report provides the first reduction to practice of the concept of nuclear transfer to produce genetically matched ES cell lines for people of different ages, states of health and sex. It is a stunning tour de force, especially when one appreciates that a mere decade ago, some considered mammalian somatic nuclear transfer nothing more than a pipe dream.

Of course, it will be many more years before cloned ES cells can be turned into routine clinical treatments for patients. From a practical standpoint, although Hwang's tenfold more efficient derivation of cloned ES cell lines is impressive, the shortage of fresh human eggs to reprogram somatic cell nuclei and derive ES cells remains a considerable drawback. Solutions may lie in the observation that oocytes 'might' be derived from human ES cells and in the as yet uncorroborated claims that ES cells themselves may be able to reprogram somatic cell nuclei-either avenue would circumvent the egg donation problem, but neither is unlikely to quell the ethical disagreements over ES cells, which continue to rage and show no signs of resolution.

Meanwhile, forward steps continue to be made in the field of adult stem cell therapy. One estimate is that there are currently over 80 therapies and around 300 clinical trials underway using such cells. Hematopoietic stem cell transplants are routine clinical practice and more than 300 patients with type I diabetes have now undergone transplants of islet cells using the so-called Edmonton protocol, with a significant proportion staying off insulin injections for several years.

But the field remains beset by problems of reproducibility. Often, the level of therapeutic benefit is minimal. Implanted cells die because of immune attack or other problems. It is commonly not clear whether cells are expanding, fusing with recipient cells or exerting an effect through secreted growth factors. Unraveling these problems will require progress on several fronts.

For ES cells it will be important to find conditions that can prevent or delay the development of chromosomal aneuploidies or epigenetic derangement (resulting in aberrant expression of imprinted genes) during extended culture. Culture protocols will need to dispense with potentially immunogenic animal components, serum and matrix proteins yet still provide sufficient quantities of desired cell types within a reasonable time frame using clinical-grade manufacturing processes.

Perhaps the most formidable challenge, though, will be to uncover the appropriate genetic, chemical and environmental cues that guide stem cells down specific developmental pathways in a temporally and spatially dependent manner-an effort that will likely occupy researchers for years, perhaps decades, to come. Systematic efforts will be required not only to identify techniques that can guarantee purity and authenticity of cell preparations destined for therapy but also fail-safe mechanisms capable of destroying any renegade cells after transplantation. It will also be essential to determine the extent to which stem cells must be differentiated in vitro before transplantation to achieve optimal therapeutic outcomes.

The behavior of cells engrafted into patients will require close monitoring. Just as malignant mouse embryonic carcinoma cells can contribute to the normal development of chimeric mice when introduced into an early embryo, conversely, stem cells can easily proliferate out of control or differentiate into inappropriate cell types if exposed to the wrong developmental cues in patients. An example of how things can go wrong was provided several years ago when a number of Parkinson patients, who had received transplants of human fetal mesencephalic dopaminergic neurons into their brains (denervated striatum), started to develop dyskinesias (jerky involuntary movements), possibly as a result of a poor distribution of dopaminergic synaptic contacts with recipient neurons. Even more grave, however, is the potential havoc that an ES cell could wreak through its propensity to form teratomas (tumors comprising a jumbled assortment of tissues). For this reason, the application of ES cells in a clinical setting will require especially stringent oversight.

That cell therapy is challenging should surprise no one. No other therapeutic modality comes close to approaching the complexity, fragility or variability of human cells. And because cells represent a potential reservoir of communicable pathogens, the difficulty of detecting pathogens and sterilizing cells presents a particular headache for regulatory agencies.

With all these caveats, it might seem prudent to shift focus to recombinant factors or small molecules that can stimulate expansion of the body's endogenous stem cells. Indeed, several biotech blockbuster drugs do just that: recombinant erythropoietin and granulocyte-macrophage colony stimulating factor replenish blood cells via expansion and differentiation of hematopoietic stem cells; recombinant thrombopoietin boosts platelet production from megakaryocytes. Whether there are sufficient endogenous reserves of stem cells to mediate repair in a given indication will depend on the target tissue and progression of disease. It seems likely, though, that reawakening the body's own repair mechanisms will be efficient only for certain indications and for tissues with an inherent regenerative capacity.

If there is one lesson that the 30 or so years of clinical experience with stem cells has taught us, it is that it is too early to leap to grand conclusions about which types of cells — whether ES or adult stem cells—will prove most useful. It is also too early to know which approaches will work in which conditions.

Transforming these therapies into routine clinical practice will require meticulous characterization and purification of donor cells, systematic study of the mechanisms by which cells integrate, form new tissues and restore function at the site of engraftment, and the implementation of rigorous diagnostic criteria to select those patients who would most benefit from therapy. Anything less will permanently relegate them to treatments of last resort. 